\title{
ON THE GLASS NUMBER OF REPRESENTATIONS OF AN ORDER
}

\author{
IRVING REINER
}

1. Introduction. We shall use the following notation throughout:

$$
\begin{aligned}
R & =\text { Dedekind ring }(5) . \\
\mathfrak{u} & =\text { multiplicative group of units in } R . \\
h & =\text { class number of } R . \\
K & =\text { quotient field of } R . \\
p & =\text { prime ideal in } R . \\
R_{p} & =\text { ring of } p \text {-adic integers in } K .
\end{aligned}
$$

We assume that $h$ is finite, and that for each prime ideal $p$, the index $(R: p)$ is finite.

Let $A$ be a finite-dimensional separable algebra over $K$, with an identity element $e$ (4, p. 115). Let $G$ be an $R$-order in $A$, that is, $G$ is a subring of $A$ satisfying

(i) $e \in G$,

(ii) $G$ contains a $K$-basis of $A$,

(iii) $G$ is a finitely-generated $R$-module.

By a $G$-module we shall mean a left $G$-module which is a finitely-generated torsion-free $R$-module, on which $e$ acts as identity operator. An $A$-module is defined analogously, replacing $R$ by $K$. We shall assume, unless otherwise stated, that $K$ is a splitting field for $A$; thus, the only possible $A$-endomorphisms of an irreducible $A$-module $X$ are the scalar multiplications $x \rightarrow \alpha x$, $x \in X$, where $\alpha \in K$.

As in (3), we may form the non-zero ideal $\mathfrak{g} \subset R$, defined as the intersection of the ideals which annihilate the one-dimensional cohomology groups $H(G, T)$, where $T$ ranges over the set of two-sided $G$-modules. (In the special case where $G=R(\Pi)$ is the group ring of a finite group $\Pi$, the ideal $\mathfrak{g}$ is the principal ideal generated by the group order $(I I: 1)$.) Let $P=\left\{p_{1}, \ldots, p_{l}\right\}$ be the set of distinct prime divisors of $\mathfrak{g}$, and set

$$
\mathfrak{g}=\prod_{p \in P} p^{\gamma(p)} .
$$

For any $G$-module $M$, let $K M$ be the $A$-module which consists of the $K$-linear combinations of the elements of $M$. If we set $A_{p}=R_{p} G$, we may likewise define the $A_{p}$-module $M_{p}=R_{p} M$. Two $G$-modules $M$ and $N$ are said to be in the same genus (notation: $M \vee N$ ) if and only if for each $p$, the modules

Received June 12,1958 . This research was supported in part by the Office of Naval Research. 
$M_{p}$ and $N_{p}$ are $A_{p}$-isomorphic. As is shown in (7), $M \vee N$ if and only if $K M \cong K N$ and $M_{p} \cong N_{p}$ for each $p \in P$.

For any $A$-module $L^{\prime}$, let $S\left(L^{\prime}\right)$ be the collection of $G$-modules $L$ for which $K L \cong L^{\prime}$. Suppose that $S\left(L^{\prime}\right)$ splits into $r_{g}$ genera, and into $r_{G}$ classes under $G$-isomorphism. As is shown in $(\mathbf{6} ; \mathbf{7}$; and $\mathbf{9})$, both $r_{g}$ and $r_{G}$ are finite. The purpose of this paper is to consider the relation between $r_{g}$ and $r_{G}$. For the special case where $L^{\prime}$ is irreducible, Maranda (7) has shown that $r_{G}=h r_{g}$. We shall restrict ourselves to the case where the irreducible constituents of $L^{\prime}$ are distinct from one another. If $L^{\prime}$ has $k$ distinct irreducible constituents, we shall prove

$$
r_{G} \geqslant h^{k} r_{g}
$$

Further, we shall show that equality holds provided that

(3) For each $\alpha \in R$ such that $(\alpha)+\mathfrak{g}=R$, there exists $\beta \in \mathfrak{u}$ for which $\beta \equiv \alpha\left(\bmod \mathfrak{g}^{\mathrm{k}-1}\right)$.

Finally, we shall obtain formulas for $r_{g}$ and $r_{G}$ in the special case where $k=2$. These formulas will show that if condition (3) fails, then $r_{G}$ may exceed $h^{2} r_{0}$ for this case.

2. Binding homomorphisms. In this section, we shall drop the hypothesis that $K$ is a splitting field for the algebra $A$. Let $L$ be a $G$-module which contains a submodule $M$, and assume that $M$ is an $R$-direct summand of $L$. Define $N=L / M$ to be the factor $G$-module. Every element of $L$ is then uniquely representable as an ordered pair $(n, m), n \in N, m \in M$, where the structure of $L$ as $R$-module is given by

$$
(n, m)+\left(n^{\prime}, m^{\prime}\right)=\left(n+n^{\prime}, m+m^{\prime}\right), \quad \alpha(n, m)=(\alpha n, \alpha m),
$$

for $n, n^{\prime} \in N, m, m^{\prime} \in M, \alpha \in R$. Further, the action of $G$ on $L$ is given by

$$
g(n, m)=\left(g n, \Lambda_{o}(n)+g m\right), \quad g \in G, \text { where } \Lambda_{o} \in \operatorname{Hom}_{R}(N, M) .
$$

Let $\Lambda: G \rightarrow \operatorname{Hom}_{R}(N, M)$ be the $R$-homomorphism defined by $g \rightarrow \Lambda_{g}$. The condition $(g h)(n, m)=g(h(n, m))$ is equivalent to

$$
\Lambda_{g h}(n)=g \Lambda_{h}(n)+\Lambda_{g}(h n), \quad g, h \in G, n \in N .
$$

Call $\Lambda \in \operatorname{Hom}_{R}\left(G, \operatorname{Hom}_{R}(N, M)\right)$ a binding homomorphism if (6) holds, and let $B(N, M)$ be the $R$-module consisting of all binding homomorphisms relative to $N, M$. The $R$ - $G$-module $L$ is then completely determined by equations (4) and (5), once an element $\Lambda \in B(N, M)$ is fixed. Let us denote this module $L$ by $(N, M ; \Lambda)$.

It is convenient to turn $\operatorname{Hom}_{R}(N, M)$ into a two-sided $G$-module $T$ by defining

$$
(g t)(n)=g(t(n)), \quad(t g) n=t(g n), \quad g \in G, n \in N, t \in \operatorname{Hom}_{R}(N, M) .
$$

We may then characterize $B(N, M)$ as the set of all $\Lambda \in \operatorname{Hom}_{R}(G, T)$ for which

$$
\Lambda_{o h}=g \Lambda_{h}+\Lambda_{g} h, \quad g, h \in G
$$


Now fix $t \in T$, and define $\Lambda \in \operatorname{Hom}_{R}(G, T)$ by

$$
\Lambda_{\imath}=g t-t g, \quad g \in G .
$$

We find readily that $\Lambda \in B(N, M)$. Let $B^{\prime}(N, M)$ be the $R$-module consisting of all the binding homomorphisms so obtained by letting $t$ range over all elements of $T$. Define the $R$-module

$$
C(N, M)=B(N, M) / B^{\prime}(N, M) .
$$

From (9) we know that $C(N, M)$ contains only finitely many elements. Furthermore, from the definition of the ideal $\mathfrak{g}$, we have

$$
\text { g. } B(N, M) \subset B^{\prime}(N, M)
$$

for any $N, M$. Finally, if $[\Lambda]$ denotes the class $\Lambda+B^{\prime}(N, M)$ of the element $\Lambda \in B(N, M)$, then we have:

$$
[\Lambda]=\left[\Lambda^{\prime}\right] \Rightarrow(N, M ; \Lambda) \cong\left(N, M ; \Lambda^{\prime}\right) .
$$

In fact, if $t \in T$ is such that $\Lambda_{o}^{\prime}-\Lambda_{g}=g t-t g, g \in G$, then the map $(n, m)$ $\rightarrow(n, m-t n)$ gives the desired isomorphism.

In the above discussion, replace $R$ by $R_{p}$. If $L^{*}$ is an $A_{p}$-module which

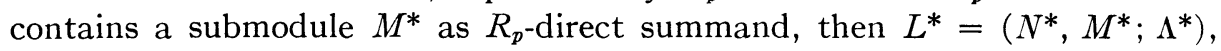
where $N^{*}=L^{*} / M^{*}$, and where

$$
\Lambda^{*}: A_{p} \rightarrow \operatorname{Hom}_{R_{p}}\left(N^{*}, M^{*}\right) .
$$

is an $R_{p}$-homomorphism satisfying $\Lambda^{*}{ }_{x y}=x \Lambda^{*} y+\Lambda^{*}{ }_{x} y, x, y \in A_{p}$. Define $B\left(N^{*}, M^{*}\right), B^{\prime}\left(N^{*}, M^{*}\right)$ and $C\left(N^{*}, M^{*}\right)$ as above. For $\Lambda^{*} \in B\left(N^{*}, M^{*}\right)$, again let $\left[\Lambda^{*}\right]=\Lambda^{*}+B^{\prime}\left(N^{*}, M^{*}\right)$. If $\gamma(p)$ is defined as in (1), we have

$$
\pi^{\gamma(p)} B\left(N^{*}, M^{*}\right) \subset B^{\prime}\left(N^{*}, M^{*}\right)
$$

where $\pi$ is an element of $p$ such that $\pi \notin p^{2}$.

Now let $N, M$ be $G$-modules, and let $N_{p}, M_{p}$ be the corresponding $A_{p^{-}}$ modules. There is a natural isomorphism of $B(N, M)$ into $B\left(N_{p}, M_{p}\right)$ which may be described as follows: for each $\Lambda \in B(N, M)$ and each $g \in G$, the map $\Lambda_{\imath} \in \operatorname{Hom}_{R}(N, M)$ may be extended in a unique manner to an element of $\operatorname{Hom}_{R_{p}}\left(N_{p}, M_{p}\right)$; we may then define $\Lambda_{x}$ for each $x \in A_{p}$ by linearity. In this way, $\Lambda$ is extended in a unique manner to an element $\Lambda^{p} \in B\left(N_{p}, M_{p}\right)$. The map $\Lambda \rightarrow \Lambda^{p}$ carries $B^{\prime}(N, M)$ into $B^{\prime}\left(N_{p}, M_{p}\right)$, and so induces an $R$-homomorphism of $C(N, M)$ into $C\left(N_{p}, M_{p}\right)$.

We may now define an $R$-homomorphism

$$
\phi: C(N, M) \rightarrow \sum_{p \in P} C\left(N_{p}, M_{p}\right)
$$

by means of

$$
\phi[\Lambda]=\left(\left[\Lambda^{p_{1}}\right], \ldots,\left[\Lambda^{p_{l}}\right]\right) .
$$


From (8), we know that $\phi$ has kernel 0 . We shall in fact show that $\phi$ is an isomorphism "onto."

THEOREM 1.

$$
C(N, M) \cong \sum_{p \in P} C\left(N_{p}, M_{p}\right)
$$

Remark. A slightly different version of this was first proved by deLeeuw (1). We shall not use the results of (8), but instead shall give a self-contained proof of the theorem.

Proof. We show firstly that the $\phi$ is an "onto" mapping. For each $p \in P$ suppose an element $\Omega^{p} \in B\left(N_{p}, M_{p}\right)$ chosen. We must prove the existence of an element $\Lambda \in B(N, M)$ such that $\left[\Lambda^{p}\right]=\left[\Omega^{p}\right], p \in P$. Let $T=\operatorname{Hom}_{R}(N, M)$, and let us set

$$
T_{p}=\operatorname{Hom}_{R_{p}}\left(N_{p}, M_{p}\right)=R_{p} \operatorname{Hom}_{R}(N, M)=R_{p} T
$$

for each prime ideal $p$.

For each $p \in P$, we may choose an element $\pi \in p$ such that $\pi \notin p^{2}$, and such that $\pi$ does not lie in any other prime ideal in the set $P$. Set

$$
a=\prod_{p \in P} \pi^{\gamma(p)}
$$

then $a \in R$, and for each $p \in P$ we may write

$$
a=\pi^{\gamma(p)} d_{p}, \quad d_{p} \in R, \quad d_{p}=\text { unit in } R_{p} .
$$

Define the integral ideal $\mathfrak{b}$ by

$$
(a)=\mathfrak{b} \cdot \prod_{p \in P} p^{\gamma(p)} .
$$

Then $\mathfrak{b}$ is not a multiple of any of the prime ideals in $P$.

We now make use of equation (8) to deduce that for each $p \in P$, there exists an element $u^{p} \in T_{p}$ such that

$$
a \cdot \Omega_{o}^{p}=g u^{p}-u^{p} g, \quad g \in G .
$$

On the other hand, $T$ is a finitely-generated $R$-module, so there exist elements $t_{1}, \ldots, t_{r} \in T$ such that

whence

$$
T=R t_{1}+\ldots+R t_{r}
$$

$$
T_{p}=R_{p} t_{1}+\ldots+R_{p} t_{r}
$$

We may therefore write (for $p \in P$ )

$$
u^{p}=\sum_{i=1}^{r} \beta_{i}^{p} t_{i}, \quad \beta_{i}^{p} \in R_{p} .
$$

Let us now choose $\alpha_{1}, \ldots, \alpha_{r} \in R$ such that

$$
\alpha_{i} \equiv \beta_{i}^{p}\left(\bmod \pi^{2 \gamma(p)} R_{p}\right), \quad p \in P, \quad \alpha_{i} \equiv 0(\bmod \mathfrak{b}) .
$$


Set

$$
t=a^{-1} \sum_{i=1}^{r} \alpha_{i} t_{i} \in K T,
$$

and define $\Lambda \in \operatorname{Hom}_{R}(G, K T)$ by

$$
\Lambda_{\sigma}=g t-t g, \quad g \in G .
$$

We shall show that this is the desired $\Lambda$, that is, $\Lambda \in B(N, M)$, and $\left[\Lambda^{p}\right]=\left[\Omega^{p}\right]$ for $p \in P$. For $p \in P$ we have

$$
a\left(\Omega_{o}^{p}-\Lambda_{g}\right)=g v^{p}-v^{p} g, \quad g \in G,
$$

where

$$
v^{p}=u^{p}-a t=\sum_{i=1}^{r}\left(\beta_{i}^{p}-\alpha_{i}\right) t_{i}
$$

From the way in which the $\alpha_{i}$ were chosen, we may therefore write

$$
v^{p}=\pi^{2 \gamma(p)} d_{p} w^{p}
$$

where $w^{p} \in T_{p}$, and thus

$$
\Omega_{g}^{p}-\Lambda_{g}=\pi^{\gamma(p)}\left(g w^{p}-w^{p} g\right), g \in G .
$$

This proves that for each $p \in P$,

$$
\Omega^{p}-\Lambda^{p} \in \pi^{\gamma(p)} B\left(N_{p}, M_{p}\right) \subset B^{\prime}\left(N_{p}, M_{p}\right),
$$

and shows incidentally that

$$
\Lambda_{0} \in T_{p}, \quad g \in G, \quad p \in P .
$$

On the other hand, we note that for each prime ideal $q \notin P$, the elements $a^{-1} \alpha_{1}, \ldots, a^{-1} \alpha_{r}$ all lie in $R_{q}$, and hence

$$
\Lambda_{\boldsymbol{\sigma}} \in T_{q}, \quad g \in G .
$$

Coupled with $(9)$, this implies that

$$
\Lambda_{\bullet} \in \bigcap_{q^{\prime}} T_{q^{\prime}}, \quad g \in G,
$$

where $q^{\prime}$ ranges over all prime ideals. The above intersection is precisely $T$, and so $\Lambda \in \operatorname{Hom}_{R}(G, T)$. That (7) holds follows at once from the definition of $\Lambda$; consequently, $\Lambda \in B(N, M)$. This completes the proof that $\phi$ is "onto."

In order to show that $\phi$ is an isomorphism, let $\Omega \in B(N, M)$ be such that $\Omega^{p} \in B^{\prime}\left(N_{p}, M_{p}\right)$ for all $p \in P$; we must show that $\Omega \in B^{\prime}(N, M)$. Since $\Omega^{p} \in B^{\prime}\left(N_{p}, M_{p}\right)$, there exists for each $p \in P$ an element $u^{p} \in T_{p}$ such that

$$
\Omega_{o}^{p}=g u^{p}-u^{p} g, \quad g \in G .
$$

By the preceding construction (with $a=1$ ), there exists $\Lambda \in B^{\prime}(N, M)$ (since now $t \in T$ ) such that

$$
\Lambda_{g}^{p} \equiv \Omega_{g}^{p}\left(\bmod \pi^{\gamma(p)} T_{p}\right), \quad g \in G .
$$


Therefore

$$
\Lambda-\Omega \in \mathfrak{g} B(N, M) \subset B^{\prime}(N, M),
$$

which shows that $\Omega \in B^{\prime}(N, M)$.

Corollary. If $N, N^{*}, M, M^{*}$ are $G$-modules such that $N \vee N^{*}$ and $M \vee M^{*}$, then $C(N, M) \cong C\left(N^{*}, M^{*}\right)$ as $R$-modules.

More generally, let

$$
L_{1} \supset L_{2} \supset \ldots \supset L_{k} \supset(0)
$$

be a set of $G$-modules such that each is an $R$-direct summand of its predecessor. Define $N_{i}=L_{i} / L_{i+1}$ to be the factor $G$-module. Then as above, every element of $L_{1}$ is uniquely representable as an ordered $k$-tuple $\left(n_{1}, \ldots, n_{k}\right)$ $n_{i} \in N_{i}$, where

$$
\begin{array}{r}
\left(n_{1}, \ldots, n_{k}\right)+\left(n_{1}^{\prime}, \ldots, n_{k}^{\prime}\right)=\left(n_{1}+n_{1}^{\prime}, \ldots, n_{k}+n_{k}^{\prime}\right), \\
\alpha\left(n_{1}, \ldots, n_{k}\right)=\left(\alpha n_{1}, \ldots, \alpha n_{k}\right)
\end{array}
$$

for $n_{i}, n_{i}{ }^{\prime} \in N_{i}, \alpha \in R$. The action of $G$ on $L_{1}$ is given by

$$
g\left(n_{1}, \ldots, n_{k}\right)=\left(g n_{1}, g n_{2}+\Lambda_{o}^{12} n_{1}, \ldots, g n_{k}+\Lambda_{o}^{1 k} n_{1}+\ldots+\Lambda_{o}^{k-1, k} n_{k-1}\right),
$$

where each $\Lambda_{0}^{i j} \in \operatorname{Hom}_{R}\left(N_{i}, N_{\jmath}\right)$, and where the $R$-homomorphisms $\Lambda^{i j}$ : $g \rightarrow \Lambda_{0}{ }^{i j}$ satisfy conditions analogous to (7). Let $B\left(N_{1}, \ldots, N_{k}\right)$ denote the set of systems $\left\{\Lambda^{i j}\right\}$ satisfying these conditions. We denote the module $L_{1}$ by the symbol $\left(N_{1}, \ldots, N_{k} ;\left\{\Lambda^{i j}\right\}\right)$.

3. Isomorphisms of modules. Throughout this section, we fix an $A$ module $L^{\prime}$ with a composition series.

$$
L=L_{1}^{\prime} \supset L_{2}^{\prime} \supset \ldots \supset L_{k}^{\prime} \supset(0),
$$

and let $N_{i}{ }^{\prime}=L_{i}{ }^{\prime} / L_{i+1}{ }^{\prime}$. We assume here that $N_{i}{ }^{\prime}$ and $N_{j}^{\prime}$ are not isomorphic for $i \neq j$, and further that $K$ is a splitting field for $A$. For any $L \in S\left(L^{\prime}\right)$, the $A$-module $K L$ will have a composition series

$$
K L=L_{1}^{\prime \prime} \supset L_{2}^{\prime \prime} \supset \ldots \supset L_{k}^{\prime \prime} \supset(0)
$$

in which $L_{i}{ }^{\prime \prime} / L_{i+1}{ }^{\prime \prime} \cong N_{i}{ }^{\prime}$. Setting $L_{i}=L_{i}{ }^{\prime \prime} \cap L$, we see that $L_{i}$ is a $G$ submodule of $L$ for which $K L_{i}=L_{i}^{\prime \prime}$. Furthermore, $L_{i+1}$ is a pure $R$-submodule of $L_{i}$, and therefore (by 5 ) is an $R$-direct summand of $L_{i}$. Put $N_{i}=$ $L_{i} / L_{\imath+1} ;$ then $K N_{i} \cong N_{i}^{\prime}$, and

$$
L=\left(N_{1}, \ldots, N_{k} ;\left\{\Lambda^{i j}\right\}\right)
$$

for some $\left\{\Lambda^{i j}\right\} \in B\left(N_{1}, \ldots, N_{k}\right)$.

Lemma 1. Let $M_{i}, N_{i}, \in S\left(N_{i}^{\prime}\right), 1 \leqslant i \leqslant k$, and suppose that

$$
\left(M_{1}, \ldots, M_{k} ;\left\{\Lambda^{i j}\right\}\right) \cong\left(N_{1}, \ldots, N_{k} ;\left\{\Omega^{i j}\right\}\right)
$$

Then $M_{i} \cong N_{i}, 1 \leqslant i \leqslant k$. 
Proof. (A modified version of this is given in (2).) It suffices to prove that if $(N, M ; \Lambda) \cong(\bar{N}, \bar{M} ; \bar{\Lambda})$, where $K N \cong K \bar{N}$ and $K M \cong K \bar{M}$, and where $K N$ and $K M$ have no common irreducible constituent, then $M \cong \bar{M}$ and $N \cong \bar{N}$. Once this is established, a simple induction argument completes the proof.

Suppose that $\theta:(N, M ; \Lambda) \cong(\bar{N}, \bar{M} ; \bar{\Lambda})$ is given by

$$
\theta(n, m)=\theta(n, 0)+\theta(0, m)=\left(\theta_{1}(n), \nu(n)\right)+\left(\mu(m), \theta_{2}(m)\right),
$$

where

$\theta_{1} \in \operatorname{Hom}_{R}(N, \bar{N}), \nu \in \operatorname{Hom}_{R}(N, \bar{M}), \mu \in \operatorname{Hom}_{R}(\mathrm{M}, \bar{N}), \theta_{2} \in \operatorname{Hom}_{R}(M, \bar{M})$.

From $\theta g(n, m)=g \theta(n, m)$ we obtain at once

$$
\begin{array}{ll}
\theta_{1} g+\mu \Lambda_{\vartheta}=g \theta_{1}, & \mu g=g \mu, \\
\bar{\Lambda}_{g} \theta_{1}+g \nu=\nu g+\theta_{2} \Lambda_{\theta}, & \theta_{2} g=\bar{\Lambda}_{\theta} \mu+g \theta_{2} .
\end{array}
$$

From (10.2) we have $\mu \in \operatorname{Hom}_{G}(M, \bar{N})$, and hence $\mu=0$, since by hypothesis $K M$ and $K \bar{N}$ have no common irreducible constituents. Equations (10.1) and (10.4) then imply that $\theta_{1} \in \operatorname{Hom}_{G}(N, \bar{N})$ and $\theta_{2} \in \operatorname{Hom}_{G}(M, \bar{M})$. Since $\theta$ is an isomorphism of $(N, M ; \Lambda)$ onto $(\bar{N}, \bar{M} ; \bar{\Lambda})$, we find readily that $\theta_{1}: N \cong \bar{N}$ and $\theta_{2}: M \cong \bar{M}$.

Lemma 2. Let $\left(N_{1}, \ldots, N_{k} ;\left\{\Lambda^{i j}\right\}\right)$ and $\left(N_{1}, \ldots, N_{k} ;\left\{\Omega^{i j}\right\}\right)$ be G-isomorphic modules in $S\left(L^{\prime}\right)$, where $N_{i} \in S\left(N_{i}{ }^{\prime}\right)$. Then there exist units $\beta_{1}, \ldots, \beta_{k} \in \mathfrak{u}$, and homomorphisms $t_{i j} \in \operatorname{Hom}_{R}\left(N_{i}, N_{j}\right)$, such that the isomorphism between these $G$-modules is given by

$$
\left(n_{1}, \ldots, n_{k}\right) \rightarrow\left(\beta_{1} n_{1}, \beta_{2} n_{2}+t_{12} n_{1}, \ldots, \beta_{k} n_{k}+t_{1 k} n_{1}+\ldots+t_{k-1},{ }_{k} n_{k-1}\right) .
$$

Proof. From the proof of the preceding lemma, we find that the isomorphism must be given by

$$
\left(n_{1}, \ldots, n_{k}\right) \rightarrow\left(\theta_{1} n_{1}, \theta_{2} n_{2}+t_{12} n_{1}, \ldots, \theta_{k} n_{k}+t_{1 k} n_{1}+\ldots+t_{k-1, k} n_{k-1}\right),
$$

with each $\theta_{i}: N_{i} \cong N_{i}$ and each $t_{i j} \in \operatorname{Hom}_{R}\left(N_{i}, N_{j}\right)$. Since $K N_{i}$ is an absolutely irreducible $A$-module, $\theta_{i}$ must be given by scalar multiplication by a unit of $R$. This completes the proof.

If $U, V$ are $R$-modules, and $f_{1}, f_{2} \in \operatorname{Hom}_{R}(U, V)$, we shall of ten abbreviate the congruence $f_{1} \equiv f_{2}\left(\operatorname{mod~g} \mathfrak{g}^{a} \operatorname{Hom}_{R}(U, V)\right)$ as $f_{1} \equiv f_{2}\left(\bmod \mathrm{g}^{a}\right)$. A similar notation will be used for $R_{p}$-modules.

Lemma 3. Let $M_{1}, \ldots, M_{k}$ be G-modules, not necessarily irreducible, and let

$$
L=\left(M_{1}, \ldots, M_{k} ;\left\{\Lambda^{i j}\right\}\right), \quad \bar{L}=\left(M_{1}, \ldots, M_{k} ;\left\{\Omega^{i j}\right\}\right)
$$

be G-modules for which

$$
\Lambda^{i j} \equiv \Omega^{i j}\left(\bmod g^{n}\right), \quad 1 \leqslant i<j \leqslant k,
$$


where $n$ is a fixed integer $\geqslant k-1$. Then there exists a G-isomorphism $\theta: L \cong \bar{L}$ such that $\theta \equiv I\left(\bmod \mathfrak{g}^{n-k+1}\right)$, where $I: L \cong \bar{L}$ is the R-isomorphism given by $\left(m_{1}, \ldots, m_{k}\right) \rightarrow\left(m_{1}, \ldots, m_{k}\right)$.

Proof. The result is trivial for $k=1$; let $k>1$, and assume the result holds at $k-1$. Let us set

$$
\begin{aligned}
& \Delta=\left(M_{2}, \ldots, M_{k} ; \Lambda^{23}, \ldots, \Lambda^{k-1, k}\right), \\
& \bar{\Delta}=\left(M_{2}, \ldots, M_{k} ; \Omega^{23}, \ldots, \Omega^{k-1, k}\right) .
\end{aligned}
$$

From the induction hypothesis we deduce the existence of a $G$-isomorphism $\theta_{1}: \Delta \cong \bar{\Delta}$ such that

$$
\theta_{1} \equiv I\left(\bmod \mathrm{g}^{n-k+2}\right) .
$$

The map $\left(m_{1}, \delta\right) \rightarrow\left(m_{1}, \theta_{1} \delta\right)$, where $m_{1} \in M_{1}, \delta \in \Delta$, then gives a $G$-isomorphism

$$
\theta_{2}:\left(M_{1}, \Delta ; \Lambda^{12}, \ldots, \Lambda^{1 k}\right)^{\prime} \cong\left(M_{1}, \bar{\Delta} ; \bar{\Lambda}^{12}, \ldots, \bar{\Lambda}^{1 k}\right)
$$

for some $\left(\bar{\Lambda}^{12}, \ldots, \bar{\Lambda}^{1 k}\right) \in B\left(M_{1}, \bar{\Delta}\right)$, and we have

$$
\theta_{2} \equiv I\left(\bmod \mathrm{g}^{n-k+2}\right) \text {. }
$$

Now set

$$
\bar{\Lambda}=\left(\bar{\Lambda}^{12}, \ldots, \bar{\Lambda}^{1 k}\right), \quad \Omega=\left(\Omega^{12}, \ldots, \Omega^{1 k}\right) .
$$

Then we see that both $\bar{\Lambda}$ and $\Omega$ are elements of $B\left(M_{1}, \bar{\Delta}\right)$, and that $\bar{\Lambda} \equiv \Omega$ $\left(\bmod \mathfrak{g}^{n-k+2}\right)$. By considering this congruence for the powers of the prime ideals dividing $\mathfrak{g}$, the method of proof of Theorem 1 shows the existence of an element $W \in \operatorname{Hom}_{R}\left(M_{1}, \bar{\Delta}\right)$ such that

$$
(\bar{\Lambda}-\Omega)_{g}=g W-W g, \quad g \in G,
$$

and where, furthermore, $W \equiv 0\left(\bmod \mathfrak{g}^{n-k+1}\right)$. The map $\left(m_{1}, \bar{\delta}\right) \rightarrow\left(m_{1}, \bar{\delta}-W m_{1}\right)$ then yields a $G$-isomorphism $\theta_{3}:\left(M_{1}, \bar{\Delta} ; \Omega\right) \cong\left(M_{1}, \bar{\Delta} ; \Lambda\right)$, where

Therefore

$$
\theta_{3} \equiv I\left(\bmod g^{n-k+1}\right) \text {. }
$$

$$
\theta_{3}^{-1} \theta_{2}:\left(M_{1}, \Delta ; \Lambda^{12}, \ldots, \Lambda^{1 k}\right) \rightarrow\left(M_{1}, \bar{\Delta} ; \Omega^{12}, \ldots, \Omega^{1 k}\right)
$$

is a $G$-isomorphism of $L$ onto $\bar{L}$ such that

$$
\theta_{3}^{-1} \theta_{2} \equiv I\left(\bmod \mathfrak{g}^{n-k+1}\right) .
$$

4. Integral classes and genera for modules with two distinct constituents. Throughout this section, we suppose that $L^{\prime}$ is an $A$-module with two distinct irreducible constituents $N^{\prime}$ and $M^{\prime}$; we assume again that $K$ is a splitting field for $A$. Let $S\left(L^{\prime}\right)$ be partitioned into $r_{G}$ classes under $G$-isomorphism, and into $r_{g}$ genera. We shall obtain formulas for $r_{G}$ and $r_{\theta}$. 
Lemma 4. Let $N \in S\left(N^{\prime}\right), M \in S\left(M^{\prime}\right)$. Then $(N, M ; \Lambda) \cong(N, M ; \bar{\Lambda})$ if and only if there exists $\beta \in \mathfrak{u}$ such that $[\bar{\Lambda}]=\beta[\Lambda]$.

Proof. From Lemma 2 we deduce the existence of units $\beta_{1}, \beta_{2} \in \mathfrak{u}$, and of $t \in \operatorname{Hom}_{R}(N, M)$, such that the isomorphism $(N, M ; \Lambda) \cong(N, M ; \bar{\Lambda})$ is given by $(n, m) \rightarrow\left(\beta_{1} n, \beta_{2} m+t n\right)$. This implies

$$
\bar{\Lambda}_{g}=\beta_{1}^{-1} \beta_{2} \Lambda_{g}+g\left(\beta_{1}^{-1} t\right)-\left(\beta_{1}^{-1} t\right) g, \quad g \in G .
$$

Setting $\beta=\beta_{1}{ }^{-1} \beta_{2}$, we have $[\bar{\Lambda}]=\beta[\Lambda]$. Conversely, starting from such a relation, we may reverse the steps to obtain an isomorphism of the modules.

Lemma 5. Let $N \in S\left(N^{\prime}\right), M \in S\left(M^{\prime}\right)$. Then $(N, M ; \Lambda) \vee(N, M ; \bar{\Lambda})$ if and only if there exists an element $\alpha \in R$ such that $(\alpha)+g=R$ and $[\bar{\Lambda}]=\alpha[\Lambda]$.

Proof. Let $(N, M ; \Lambda) \vee(N, M ; \bar{\Lambda})$. As in the preceding proof, we deduce that for each $p \in P$, there exists an element $\alpha_{p}$ which is a unit in $R_{p}$ such that the classes $\left[\Lambda^{p}\right]$ and $\left[\bar{\Lambda}^{p}\right]$ in $C\left(N_{p}, M_{p}\right)$ are related by

$$
\left[\bar{\Lambda}^{p}\right]=\alpha_{p}\left[\Lambda^{p}\right]
$$

Choose $\alpha \in R$ such that $\alpha \equiv \alpha_{p}\left(\bmod p^{\gamma(p)}\right)$ for each $p \in P$; then $(\alpha)+\mathfrak{g}=R$. Furthermore, $\left(\alpha-\alpha_{p}\right) B\left(N_{p}, M_{p}\right) \subset B^{\prime}\left(N_{p}, M_{p}\right)$, so that

$$
\alpha\left[\Lambda^{p}\right]=\alpha_{p}\left[\Lambda^{p}\right], \quad p \in P .
$$

Therefore $\left[\bar{\Lambda}^{p}\right]=\left[\alpha \Lambda^{p}\right]$ for all $p \in P$, and so by Theorem 1 we have $[\bar{\Lambda}]=$ $[\alpha \Lambda]=\alpha[\Lambda]$.

Suppose now that $S\left(N^{\prime}\right)$ splits into $\nu$ genera; according to (7), each genus splits into $h$ classes under $G$-isomorphism. Let us choose representatives of the $h \nu$ classes, say $\left\{N_{j}{ }^{i}: 1 \leqslant i \leqslant \nu, 1 \leqslant j \leqslant h\right\}$, so that all the modules with the same subscript lie in the same genus. Likewise choose representatives $\left\{M_{j}{ }^{i}: 1 \leqslant i \leqslant \mu, 1 \leqslant j \leqslant h\right\}$ of the $h \mu$ classes into which $\mathrm{S}\left(M^{\prime}\right)$ splits. Let $(N, M ; \Gamma) \in S\left(L^{\prime}\right)$, and suppose $N \vee N_{1}{ }^{i}, M \vee M_{1}{ }^{j}$. Then for each $p \in P$, there exists an element

$$
\Omega^{p} \in B\left(\left(N_{1}^{i}\right)_{p},\left(M_{1}^{j}\right)_{p}\right)
$$

such that

$$
\left(N_{p}, M_{p} ; \Gamma^{p}\right) \cong\left(\left(N_{1}^{i}\right)_{p},\left(M_{1}^{j}\right)_{p} ; \Omega^{p}\right)
$$

as $A_{p}$-modules. By Theorem 1 , there exists $\Lambda \in B\left(N_{1}{ }^{i}, M_{1}{ }^{j}\right)$ such that $\left[\Lambda^{p}\right]=\left[\Omega^{p}\right]$ for all $p \in P$. Therefore

$$
(N, M ; \Gamma) P \cong\left(N_{1}^{i}, M_{1}^{j} ; \Lambda\right)_{p}, \quad p \in P,
$$

and so

$$
(N, M ; \Gamma) \vee\left(N_{1}^{i}, M_{1}^{j} ; \Lambda\right)
$$


Hence, every module in $S\left(L^{\prime}\right)$ is in the same genus as $\left(N_{1}{ }^{i}, M_{1}{ }^{j} ; \Lambda\right)$ for some choice of $i$ and $j$ and some $\Lambda \in B\left(N_{1}{ }^{i}, M_{1}{ }^{j}\right)$. Further,

$$
\left(N_{1}^{i}, M_{1}^{j} ; \Lambda\right) \vee\left(N_{1}^{i^{\prime}}, M_{1}^{j^{\prime}} ; \Lambda^{\prime}\right)
$$

implies, by the method of proof of Lemma 1 , that $i=i^{\prime}$ and $j=j^{\prime}$. Let us set

$$
H_{i j}=\left\{\left(N_{1}^{i}, M_{1}^{j} ; \Lambda\right): \Lambda \in B\left(N_{1}^{i}, M_{1}^{j}\right)\right\}, 1 \leqslant i \leqslant \nu, 1 \leqslant j \leqslant \mu,
$$

and suppose that $H_{i j}$ splits into $r_{i j}$ genera. Then we have at once

$$
r_{g}=\sum_{i, j} r_{i j}
$$

On the other hand, any module in $S\left(L^{\prime}\right)$ is $G$-isomorphic to $\left(N_{\rho}{ }^{i}, M_{\sigma}{ }^{j} ; \Lambda\right)$ for some $i, j, \rho, \sigma$ and $\Lambda$. Further, by Lemma 1 , two such modules cannot be isomorphic unless they have the same set of indices $i, j, \rho, \sigma$. Let us set

$$
S(i, \rho ; j, \sigma)=\left\{\left(N_{\rho}^{i}, M_{\sigma}^{j} ; \Lambda\right): \Lambda \in B\left(N_{\rho}^{i}, M_{\sigma}^{j}\right)\right\},
$$

and suppose that $S(i, \rho ; j, \sigma)$ splits into $s(i, \rho ; j, \sigma)$ classes. Then

$$
r_{G}=\sum_{i, j, \rho, \sigma} s(i, \rho ; j, \sigma) .
$$

However, Lemma 4 states that $\left(N_{\rho}{ }^{i}, M_{\sigma}{ }^{j} ; \Lambda\right) \cong\left(N_{\rho}{ }^{i}, M_{\sigma}{ }^{j} ; \bar{\Lambda}\right)$ if and only if there exists $\beta \in \mathfrak{u}$ such that $[\bar{\Lambda}]=\beta[\Lambda]$. Furthermore, the Corollary to Theorem 1 shows that $C\left(N_{\rho}{ }^{i}, M_{\sigma}{ }^{j}\right)$ is (as $R$-module) independent of $\rho$ and $\sigma$. Therefore $s(i, \rho ; j, \sigma)=s(i, 1 ; j, 1)$ for all $\rho$ and $\sigma$, and we have

$$
r_{G}=h^{2} \sum_{i, j} s_{i j}
$$

where $s_{i j}=s(i, 1 ; j, 1)$ is the number of classes into which $H_{i j}$ splits.

Before proceeding with the calculation of $r_{i j}$ and $s_{i j}$, it will be convenient to introduce some notations. For a non-zero ideal $\mathfrak{a}$ in $R$, let $\phi(\mathfrak{a})$ denote the number of residue classes in $R / \mathfrak{a}$ which are relatively prime to $\mathfrak{a}$. If $\mathfrak{a}+\mathfrak{b}=R$, then $\phi(\mathfrak{a} \mathfrak{b})=\phi(\mathfrak{a}) \phi(\mathfrak{b})$. Next, let $u(\mathfrak{a})$ denote the number of distinct residue classes in $(\mathfrak{u}+\mathfrak{a}) / \mathfrak{a}$; of course, $u(\mathfrak{a})$ is a divisor of $\phi(\mathfrak{a})$. However, $u(\mathfrak{a})$ is not a multiplicative function of $\mathfrak{a}$, as is seen from the example where $K$ is the rational field.

Lemma 6. Let $N \in S\left(N^{\prime}\right), M \in S\left(M^{\prime}\right)$, and $H=\{(N, M ; \Lambda): \Lambda \in B(N, M)\}$. Suppose $H$ splits into $r$ genera and $s$ classes. Let $d(\mathfrak{a})$ be the number of elements in $C(N, M)$ with order ideal a. Then

$$
r=\sum_{\mathfrak{a}} d(\mathfrak{a}) / \phi(\mathfrak{a}), \quad s=\sum_{\mathfrak{a}} d(\mathfrak{a}) / u(\mathfrak{a}),
$$

both sums extending over all divisors of $\mathfrak{g}$.

(The order ideal of an element $c \in C(N, M)$ is $\{\alpha \in R: \alpha c=0\}$.) 
Proof. Let us use the symbol $(N, M ; c)$ to denote the collection of mutually isomorphic modules $\{(N, M ; \Lambda): \Lambda \in c\}$, where $c \in C(N, M)$. By Lemma 4, $(N, M ; c)$ and $\left(N, M ; c^{\prime}\right)$ cannot lie in the same genus unless $c$ and $c^{\prime}$ have the same order ideal. Consider the set of $d(\mathfrak{a})$ elements of $C(N, M)$ with given order ideal $a$. For a fixed $c$ in this set, all those $c^{\prime}$ of the form $\alpha c$, where $\alpha \in R$ is such that $(\alpha)+\mathfrak{g}=R$, will yield modules in the same genus as those obtained from $c$. But as $\alpha$ ranges over all elements of $R$ for which $(\alpha)+\mathfrak{g}=R, \alpha c$ gives exactly $\phi(\mathfrak{a})$ distinct elements of $C(N, M)$. Therefore

$$
r=\sum_{\mathfrak{a}} d(\mathfrak{a}) / \phi(\mathfrak{a}) .
$$

A similar argument gives the formula for $s$.

Let $d_{p}\left(p^{n}\right)$ denote the number of elements in $C\left(N_{p}, M_{p}\right)$ having order ideal $p^{n}$. Then

$$
d_{p}\left(p^{n}\right)=\tau\left(p^{n}\right)-\tau\left(p^{n-1}\right),
$$

where $\tau\left(p^{n}\right)$ denotes the number of elements of $C\left(N_{p}, M_{p}\right)$ which are annihilated by $p^{n}$. From Theorem 1,

$$
d(\mathfrak{a})=\prod_{p \in P} d_{p}\left(p^{a(p)}\right), \text { where } \mathfrak{a}=\prod_{p \in P} p^{a(p)} .
$$

We may therefore write

$$
r=\prod_{p \in P}\left\{\sum_{a=0}^{\gamma(p)} d_{p}\left(p^{a}\right) / \phi\left(p^{a}\right)\right\}
$$

which confirms the result in (7) that the number of genera is the product over all $p \in P$ of the number of classes into which $S\left(L^{\prime}\right)$ splits under $A_{p}{ }^{-}$ isomorphism. The corresponding multiplicative formula for $s$ fails to hold, because $u(\mathfrak{a})$ is not multiplicative.

Applying Lemma 6 to our original problem, we may summarize our result as follows.

Theorem 2. Let $N^{1}, \ldots, N^{v}$ be representatives of the genera into which $S\left(N^{\prime}\right)$ splits, and $M^{1}, \ldots, M^{\mu}$ representatives of the genera of $S\left(M^{\prime}\right)$. For each divisor a of $\mathfrak{g}$, let $d_{i j}(\mathfrak{a})$ denote the number of elements in $C\left(N^{i}, M^{j}\right)$ having order ideal a. Then $S\left(L^{\prime}\right)$ splits into $r_{g}$ genera and $r_{G}$ classes, where

$$
r_{g}=\sum_{\mathfrak{a}} \sum_{i, j} d_{i j}(\mathfrak{a}) / \phi(\mathfrak{a}), \quad r_{G}=h^{2} \sum_{\mathfrak{a}} \sum_{i, j} d_{i j}(\mathfrak{a}) / u(\mathfrak{a}) .
$$

Here, $\phi(\mathfrak{a})$ is the number of residue classes in $R / \mathfrak{a}$ which are relatively prime to $\mathfrak{a}$, and $u(\mathfrak{a})$ is the number of distinct elements of $(\mathfrak{u}+\mathfrak{a}) / \mathfrak{a}$.

Corollary. We have $r_{G} \geqslant h^{2} r_{g}$, with equality provided that $\phi(\mathfrak{g})=u(\mathfrak{g})$. Furthermore, if any $C\left(N^{i}, M^{j}\right)$ contains an element of order ideal $\mathfrak{a}$, where $u(\mathfrak{a})<\phi(\mathfrak{a})$, then $r_{G}>h^{2} r_{g}$. 
5. Integral classes and genera in the general case. Now let $L^{\prime}$ be an $A$-module with $k$ distinct irreducible constituents, and let $K$ be a splitting field for $A$. We preserve the notation introduced at the beginning of $\S 3$. In this section we shall generalize the results given in the Corollary to Theorem 2 .

For each $\kappa(1 \leqslant \kappa \leqslant k)$, let $\left\{{N_{\kappa}}^{i j}: 1 \leqslant i \leqslant \nu(\kappa), 1 \leqslant j \leqslant h\right\}$ be a full set of representatives of the $h \nu(\kappa)$ classes into which the set $\mathrm{S}\left(N_{\kappa}{ }^{\prime}\right)$ splits; suppose these representative modules are so chosen that modules with the same indices $i$ and $\kappa$ lie in the same genus. Then every module in $S\left(L^{\prime}\right)$ is of the form

$$
\left(N_{1}^{i_{1} j_{1}}, \ldots, N_{k}^{i_{k} j_{k}} ;\left\{\Lambda^{i j}\right\}\right) .
$$

Let $S\left(i_{1}, j_{1} ; \ldots ; i_{k}, j_{k}\right)$ be the set of all such modules obtained by letting $\left\{\Lambda^{i j}\right\}$ range over all systems in

$$
B\left(N_{1}^{i_{1} j_{1}}, \ldots, N_{k}^{i_{k} j_{k}}\right) \text {, }
$$

and let this set split into $r\left(i_{1}, j_{1} ; \ldots ; i_{k}, j_{k}\right)$ genera and $s\left(i_{1}, j_{1} ; \ldots ; i_{k}, j_{k}\right)$ classes. From the Corollary to Theorem 1 , we see that $r\left(i_{1}, j_{1} ; \ldots ; i_{k}, j_{k}\right)$ is independent of $\left(j_{1}, \ldots, j_{k}\right)$, and therefore

$$
r_{o}=h^{-k} \sum r\left(i_{1}, j_{1}, \ldots ; i_{k}, j_{k}\right), \quad r_{G}=\sum s\left(i_{1}, j_{1}, \ldots ; i_{k}, j_{k}\right),
$$

both summations extending over all possible values of the $i$ 's and $j$ 's. This implies the result that

Finally, we prove:

$$
r_{G} \geqslant h^{k} r_{g}
$$

Theorem 3. If $u\left(\mathrm{~g}^{k-1}\right)=\phi\left(\mathrm{g}^{k-1}\right)$, then $r_{G}=h^{k} r_{g}$.

Proof. We remark that the hypothesis of the Theorem is simply a restatement of condition (3) given in the introduction. To prove the theorem, we need only show that $r\left(i_{1}, j_{1} ; \ldots ; i_{k}, j_{k}\right)=s\left(i_{1}, j_{1} ; \ldots ; i_{k}, j_{k}\right)$. We simplify the notation by letting $M_{\kappa} \in \mathrm{S}\left(N_{\kappa}{ }^{\prime}\right), 1 \leqslant \kappa \leqslant k$. We shall prove that if

$$
L=\left(M_{1}, \ldots, M_{k} ;\left\{\Lambda^{i j}\right\}\right), \quad \bar{L}=\left(M_{1}, \ldots, M_{k} ;\left\{\bar{\Lambda}^{i j}\right\}\right)
$$

are such that $L \vee \bar{L}$, then also $L \cong \bar{L}$.

Since $L_{p} \cong \bar{L}_{p}$ for each $p \in P$, Lemma 2 shows the existence of units $\beta_{1}{ }^{p}, \ldots, \beta_{k}{ }^{p}$ in $R_{p}$, and homomorphisms

$$
t_{i j}^{p} \in \operatorname{Hom}_{p}\left(\left(M_{i}\right)_{p},\left(M_{j}\right)_{p}\right)
$$

such that the isomorphism $L_{p} \cong \bar{L}_{p}$ is given by

$$
\left(m_{1}, \ldots, m_{k}\right) \rightarrow\left(\beta_{1}^{p} m_{1}, \beta_{2}^{p} m_{2}+t_{12}^{p} m_{1}, \ldots, \beta_{k}^{p} m_{k}+t_{1 k}^{p} m_{1}+\ldots+t_{k-1, k}^{p} m_{k-1}\right) .
$$

By the hypothesis of the theorem, we may choose units $\beta_{1}, \ldots, \beta_{k} \in \mathfrak{u}$ such that

$$
\beta_{\kappa} \equiv \beta_{\kappa}^{p} \quad\left(\bmod p^{(k-1) \gamma(p)}\right), \quad p \in P, \quad 1 \leqslant \kappa \leqslant k .
$$


As in the proof of Theorem 1, we may choose homomorphisms $w_{i j} \epsilon$ $\operatorname{Hom}_{R}\left(M_{i}, M_{j}\right)$ such that

$$
w_{i j}^{p} \equiv t_{i j}^{p} \quad \bmod p^{(k-1) \gamma(p)}, \quad 1 \leqslant i<j \leqslant k, \quad p \in P .
$$

Then the map

$$
\left(m_{1}, \ldots, m_{k}\right) \rightarrow\left(\beta_{1} m_{1}, \beta_{2} m_{2}+w_{12} m_{1}, \ldots, \beta_{k} m_{k}+w_{1 k} m_{1}+\ldots+w_{k-1, k} m_{k-1}\right)
$$

gives a $G$-isomorphism of $L$ onto a module $L^{*}$ where $L^{*}=\left(M_{1}, \ldots, M_{k} ;\left\{\Omega^{i j}\right\}\right)$ and $\Omega^{i j} \equiv \bar{\Lambda}^{i j}\left(\bmod \mathrm{g}^{k-1}\right)$ for $1 \leqslant i<j \leqslant k$. By Lemma 3 we then have $L^{*} \cong \bar{L}$, which completes the proof of the theorem.

It would be of interest to obtain formulas for $r_{G}$ and $r_{g}$ which generalize those given in Theorem 2 .

\section{REFERENCES}

1. K. deLeeuw, Some applications of cohomology to algebraic number theory and group representations, unpublished.

2. F. E. Diederichsen, Ueber die Ausreduktion ganzzahliger Gruppendarstellungen bei arithmetischer Äquivalenz, Hamb. Abh., 14 (1938), 357-412.

3. D. G. Higman, On orders in separable algebras, Can. J. Math., 7 (1955), 509-515.

4. N. Jacobson, The theory of rings (New York, 1943).

5. I. Kaplansky, Modules over Dedekind rings and valuation rings, Trans. Amer. Math. Soc., $7(1952), 327-40$.

6. J.-M. Maranda, On p-adic integrab representations of finite groups, Can. J. Math., 5 (1953), 344-355.

7. - On the equivalence of representations of finite groups by groups of automorphisms of modules over Dedekind rings, Can. J. Math., 7 (1955), 516-526.

8. I. Reiner, Maschke modules over Dedekind rings, Can. J. Math., 8 (1956), 329-334.

9. H. Zassenhaus, Neuer Beweis der Endlichkeit der Klassenzahl bei unimodularer Äquivalenz endlicher ganzzahliger Substitutionsgruppen, Hamb. Abh., 12 (1938), 276-288.

University of Illinois 